\title{
A novel approach to improving coagulation sample ordering in an emergency department
}

\author{
Emma Murphy, Sile MacGlone, Claire McGroarty \\ United Kingdom
}

\begin{abstract}
Driven by emergency department targets, there is a need for rapid initial assessment and investigations of attendees to the department, and blood tests are often performed before full patient assessment. It has been shown that many investigations ordered in the emergency department are inappropriate. Coagulation samples are acknowledged as one the commonest blood samples requested on admission. We predicted that the majority of the routine coagulation samples performed in our ED department were unnecessary.
\end{abstract}

We aimed to determine if coagulation tests sent from our department were appropriate, develop guidance for appropriate testing and to increase the percentage of appropriate tests to $90 \%$. Criterion based audit was used. All coagulation samples sent from the ED over a one week period were reviewed and the indications for testing compared to guidance developed by consensus with ED consultants.

On the first data collection, 66 of $369(17 \%)$ samples were deemed appropriate. Feedback to clinical staff was given at educational meetings and appropriate indications discussed. In collaboration with both senior nursing and medical staff, coagulation screen request bottles were removed from the main clinical area and were only available in the resuscitation area.

Following these interventions, 69 of $97(71 \%)$ samples were deemed appropriate and a further intervention is planned to reach our standard.

This improvement could lead to a $£ 100,000$ saving annually and a cross-site collaborative study is planned to spread these improvements.

\section{Problem}

The number of attendances to emergency departments (ED) in Scotland has continued to rise since $2008 / 09$, with a $3.1 \%$ increase to 1.62 million attendees in 2012/13.[1] As the number of attendances increase, the requesting of routine investigations will proportionally increase.

Driven by emergency department targets, there is a need for rapid initial assessment and investigations of attendees to the department and as a consequence blood tests are often performed before full patient assessment.

Following the introduction of a new electronic laboratory requesting system in the Greater Glasgow and Clyde health board, coagulation screens are available as a routine test. No standard proforma exists for coagulation screen requesting in our busy emergency department based at the Western Infirmary, Glasgow. We predicted that the majority of the routine coagulation samples performed in our ED department were unnecessary

\section{Background}

It has been shown that many investigations ordered in the emergency department are inappropriate.[2-4] Coagulation samples are acknowledged as one the commonest blood samples requested on admission.[2,5]
The common expectation among doctors is that these results will assist in identifying patients who are at increased risk of bleeding. However, there are many pitfalls of a coagulation screen and a normal result can be found in the Von Willebrand's disease, the commonest of the inherited bleeding disorders. Coagulation screens do not provide any information regarding fibrinolysis or platelet dysfunction.

Various studies have shown that coagulation studies have limited value prior to surgery and invasive procedures and have suggested a structured bleeding history is of more value in identifying those who require further evaluation.[6-8]

Similar studies performed in medical assessment units and emergency department have advocated the use of coagulation testing policy to guide staff in the requesting of these samples.[5,9] A study carried out in a Glasgow ED removed coagulation bottles from the main clinical area reducing the number of samples sent.

\section{Baseline measurement}

The haematology laboratory at our hospital provided data of all coagulation samples sent from the ED over a one week period between March 24th and 31st 2014. In collaboration with the ED consultants, we set out indications for appropriate requesting of coagulation screens in the ED (Appendix 1).

We used this to analyse the coagulation screen results sent over a 
one week period to determine the reason for request, grade of requestor, speciality of requestor, and whether the result was normal or abnormal. This information was gathered from a variety of sources including the electronic patient record (clinical portal), electronic laboratory requesting system (Trakcare) and electronic drug summaries (ECS).

The number of coagulation samples sent over the initial one week period totalled 369 samples of which 66 samples (17.9\%) were deemed appropriate. The ED doctors were responsible for the largest number of coagulation requests but the specialities requested a higher proportion of inappropriate samples (table 1). Of the 56 speciality requests, ED nurses requested 34 samples of which two $(5.9 \%)$ were appropriate.

Of the samples requested 33 had an abnormal result of which 24 (72.7\%) were on warfarin. Each coagulation sample costs $£ 6$ at a cost of £2214 in a one week period, of which £396 was appropriate.

See supplementary file: ds4372.doc - "Table 1"

\section{Design}

The results were fed back to both nursing and medical staff through a nursing education session and at the daily ED handovers. The appropriate indications were set out to staff at these sessions.

It was felt that the majority of coagulation screens were being performed as a routine test as the coagulation sampling bottles were readily available in the department. As a result it was decided the coagulation screen request bottles should be removed from the main clinical area and only be available in the resuscitation area. A poster was displayed at the main clinical work station reminding staff that coagulation screens were not routine and set out the indications for appropriate requests. The aim of removing the samples was to encourage staff to think about why they were requesting the sample and not view it as a routine admission blood test.

It was decided to perform a criterion based audit and use the data to drive improvement and efficiency. Our criterion was requests for a coagulation screen should only be performed for the indications contained in appendix 1 . A standard of $90 \%$ was set and we hoped to achieve this within three months.

\section{Strategy}

PDSA cycle 1: Nursing and medical staff were asked to complete a questionnaire indicating the reasons for requesting of coagulation samples in the ED. The answers from the nursing staff highlighted that they had a limited knowledge of the appropriate indications. The answers from medical staff highlighted that they were aware of the appropriate indications. A nursing staff education session was held within the department to target this.

PDSA cycle 2: A set of indications for routine coagulation ordering within the department were designed and distributed to senior medical staff within the department. Feedback was sought regarding the outlined indications. Following discussions with senior medical staff, the coagulation screen indications were re-designed.

PDSA cycle 3: In collaboration with both senior nursing and medical staff, it was decided the coagulation screen request bottles should be removed from the main clinical area and only be available in the resuscitation area.

PDSA cycle 4: The coagulation samples were restocked in the main department on two separate occasions. This was due to a change in nursing support staff who were not aware of the new policy. This was rectified in collaboration with senior medical and nursing staff who were able to highlight this at the daily nursing handover during the initial period of change.

PDSA cycle 5: The intervention results were monitored over a one week period, four weeks after the intervention.

\section{Results}

The haematology laboratory provided data of all coagulation samples sent from 12th to 19th May 2014, four weeks after the intervention. We used the coagulation indication proforma to analyse the coagulation screen results sent over a one week period to determine the reason for request, grade of requestor, speciality of requestor, and whether the result was normal or abnormal. This information was gathered from a variety of sources including the electronic patient record (clinical portal), electronic laboratory requesting system (Trakcare) and electronic drug summaries (ECS).

Following the removal of coagulation bottles from the main department, 97 coagulation screens were requested of which 69 (71.1\%) were appropriate. The ED doctors remained the largest requestor of all coagulations samples, but the specialities again requested a significantly higher proportion of inappropriate samples (table 2). The specialties requested only $20.1 \%$ of all samples sent but were responsible for three quarters of the inappropriate samples sent. Of the 20 speciality requests, ED nurses requested four coagulation screens which were all appropriate.

Of the samples requested, 31 were abnormal of which six (19.4\%) were on warfarin. The cost of coagulation samples in a week period totalled £582, of which £414 was appropriate.

See supplementary file: ds4371.doc - "Table 2"

\section{Lessons and limitations}

Introducing change to the emergency department proved to be challenging at times. Due to the high number of staff from various specialities, working in the department, it was difficult to implement change. When the coagulation samples were initially removed from the department, they were re-stocked in the main clinical area on two separate occasions. This was due to a change in nursing support staff who were not aware of the new policy. This was 
rectified in collaboration with senior medical and nursing staff who were able to highlight this at the daily nursing handover during the initial period of change.

The second set of data highlighted that the specialities working the emergency department did not adhere to the new policy ordering. The doctors working in the specialities do not partake in the department handovers and as such may not have been aware of the changes. In order to improve the results further, the specialty doctors and their clinical leads will need to be involved.

This study was performed over a relatively short period of time and as such focussed on the same cohort of medical and nursing staff working in the department. Medical staff rotate through the emergency department every four to six months and as such the new standard will need to be maintained by senior medical and nursing staff who remain in the department throughout these transitions.

The removal of the coagulation bottles from the main clinical area resulted in a change to a longstanding routine in the department and as such more reinforcement may be required to ensure this change is maintained. A repeat audit would need to be performed to ensure the sustainability of the change and ensure our $90 \%$ target is achieved.

\section{Conclusion}

Through a simple change in longstanding clinical practice, we were able to reduce the number of unnecessary coagulation samples sent from the emergency department. The number of appropriate samples improved from $17 \%$ to $71 \%$ with a simple intervention. Without the above intervention, the cost of coagulation samples would total $£ 115,128$ per year. Following the intervention, our aim is for $90 \%$ of coagulations sample to be appropriate resulting in a saving of almost $£ 100,000$ over a one year period.

In an attempt to improve our results further, we are planning on changing the laboratory interface available within our department and as a result coagulation samples will not appear as a routine admission blood test. Other ED departments within our health board have shown interest and a cross-site collaborative study has been proposed.

\section{References}

1. Audit Scotland 2014. Accident and Emergency: performance update. [cited 14 July 2014] Edinburgh:

www.audit-

scotland.gov.uk/docs/health/2014/nr 140508 ae update.pdf

1. Rehmani R, Amanullah A. Analysis of blood tests in emergency department of a tertiary care hospital. Postgrad Med J 1999; 75: 662-6.

2. Young DW. Improving laboratory usage: a review. Postgrad Med J 1988; 64: 283-9.
3. Sandler G. Do emergency tests help in the management of acute medical admissions? BMJ 1984; 289: 973-7.

4. Mainwaring CJ, Chana J, Evans J. Best practice in audit: Audit of coagulation screen requests from patients admitted to the Medical assessment Unit. London: The Royal College of Pathologists, 2007.

5. Kaplan EB, Sheiner LB, Boeckmann AJ et al. The usefulness of pre-operative laboratory screening. JAMA 1985; 253: 3576-81.

6. Chee YL, Crawford JC, Watson HG et al. Guidelines on the assessment of bleeding risk prior to surgery or invasive procedures. British Committee for Standards in Haematology. Br J Haematol 2008; 140: 496-504.

7. Kozak EA, Brath LK. Do screening coagulation tests predict bleeding in patients undergoing fiberoptic bronchopscopy? Chest 1994; 106: 703-5.

8. Martin D, Beardsell. Is routine coagulation testing necessary in patients presenting to the emergency department with chest pain? Emerg Med J 2012; 29: 184-7.

\section{Declaration of interests}

Nothing to declare.

\section{Acknowledgements}

Dr Susan Daisley, Dr Duncan McNab, Dr John McKay, Paul Bowie. 